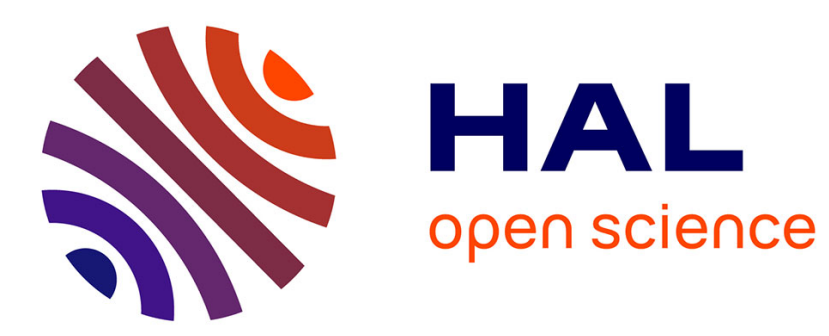

\title{
Voxel-based attribute profiles on lidar data for land cover mapping
}

Florent Guiotte, Sébastien Lefèvre, Thomas Corpetti

\section{To cite this version:}

Florent Guiotte, Sébastien Lefèvre, Thomas Corpetti. Voxel-based attribute profiles on lidar data for land cover mapping. IEEE International Geosciences and Remote Sensing Symposium (IGARSS), 2019, Yokohama, Japan. hal-02343963

\section{HAL Id: hal-02343963 https://hal.science/hal-02343963}

Submitted on 13 Nov 2019

HAL is a multi-disciplinary open access archive for the deposit and dissemination of scientific research documents, whether they are published or not. The documents may come from teaching and research institutions in France or abroad, or from public or private research centers.
L'archive ouverte pluridisciplinaire HAL, est destinée au dépôt et à la diffusion de documents scientifiques de niveau recherche, publiés ou non, émanant des établissements d'enseignement et de recherche français ou étrangers, des laboratoires publics ou privés. 


\section{VOXEL-BASED ATTRIBUTE PROFILES ON LIDAR DATA FOR LAND COVER MAPPING}

Florent Guiotte

LETG-Rennes, IRISA

Univ. Rennes 2

florent.guiotte@univ-rennes2.fr
Sébastien Lefèvre

IRISA

Univ. Bretagne Sud

sebastien.lefevre@irisa.fr
Thomas Corpetti

LETG-Rennes

CNRS

thomas.corpetti@univ-rennes2.fr

\begin{abstract}
This paper deals with strategies for LiDAR data analysis. While a large majority of studies first rasterize 3D point clouds onto regular $2 \mathrm{D}$ grids and then use 2D image processing tools for characterizing data, our work rather suggests to keep as long as possible the $3 \mathrm{D}$ structure by computing features on $3 \mathrm{D}$ data and rasterize later in the process. By this way, the vertical component is still taken into account. In practice, a voxelization step of raw data is performed in order to exploit mathematical tools defined on regular volumes. More precisely, we focus on attribute profiles that have been shown to be very efficient features to characterize remote sensing scenes. They require the computation of an underlying hierarchical structure (through a Max-Tree). Experimental results obtained on urban LiDAR data classification support the performances of this strategy compared with an early rasterization process.
\end{abstract}

Index Terms - LiDAR, land cover mapping, attribute profiles, max-tree, multiscale representation, voxelization

\section{INTRODUCTION}

Since a decade, LiDAR acquisitions are more and more exploited in a large variety of fields such as geosciences (flow, erosion, rock deformations, ...), computer graphics (3D reconstruction), urban environments analysis and of course Earth Observation (detection of trees, roads, buildings, ... ).

Unlike images defined on regular 2D grids for which a large number of computer vision and image processing techniques are available, the non regular domain on which $3 \mathrm{D}$ point clouds are defined requires the conception of alternative and dedicated approaches. In addition, the multi-scale aspect of structures embedded in 3D LiDAR point clouds calls for the use of multi-scale techniques.

For that reason, many authors first project 3D data onto 2D regular grids (the so-called "rasterization" process) and then exploit the large panel of existing multi-scale tools. For example authors of [1] perform the segmentation and classification of urban point clouds with mathematical morphology after a projection of raw data on digital elevation model (DEM), and then re-project the results on point clouds. The same idea is used in [2] where a series of attribute profiles are computed on 2D grids containing various information related extracted during the mapping from 3D to $2 \mathrm{D}$ (number of points in a cell, first echo, last echo, ....).

Despite relevant results, the direct projection of raw data on a 2D grid prevents us from analysing into details the complete 3D structures. Conversely, it is possible to analyze the $3 \mathrm{D}$ point clouds by computing dedicated 3D features and then project these structures

The authors acknowledge the support of Région Bretagne (CAMLOT doctoral project).

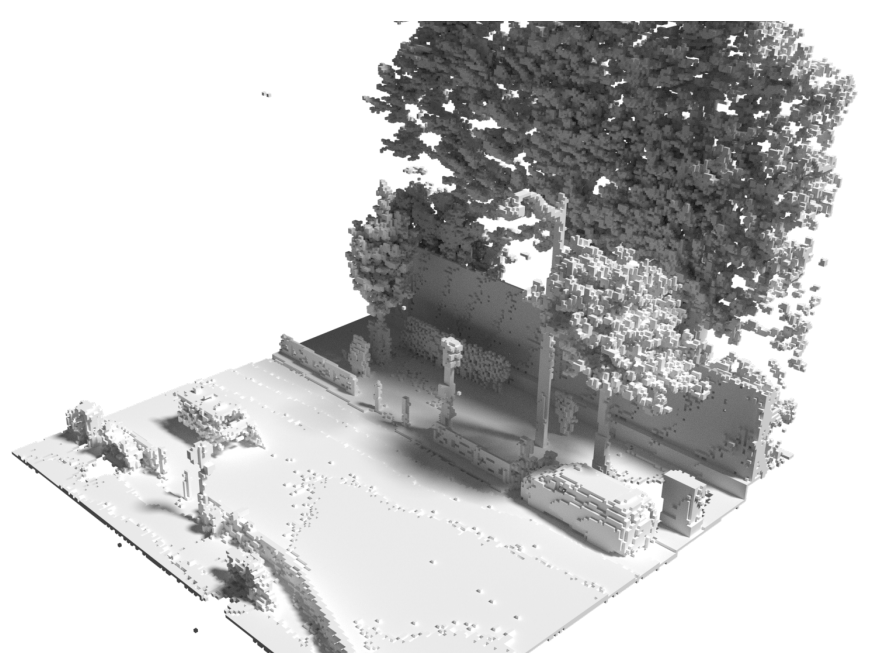

Fig. 1: LiDAR data represented within $3 D$ voxel space: one can notice the information richness contained in the vertical distribution which is usually lost during the rasterization phase.

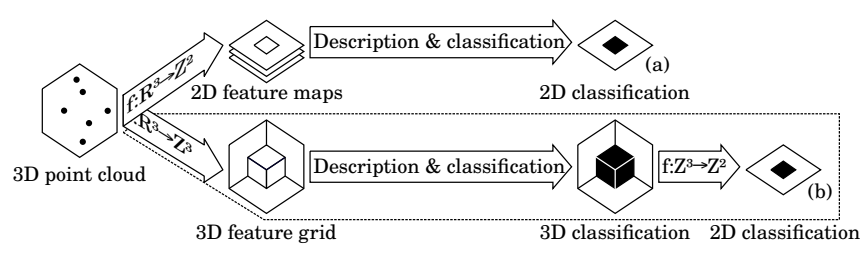

Fig. 2: Different LiDAR data classification approaches: (a) usual methods with 2D rasterization process beforehand, (b) proposed method with postponed $2 \mathrm{D}$ rasterization.

on $2 \mathrm{D}$ regular grids. We explore such strategy in this paper, since it provides rich $3 \mathrm{D}$ features as shown in Figure 1. For the sake of illustration, we focus here on attribute profiles that are popular feature in remote sensing. These features are extracted through morphological hierarchies that provide a rich and efficient multi-scale analysis framework. The overall process is schematized in Figure 2.

\subsection{Attribute Profiles}

Morphological attribute profiles are well-established pixel features that embed spatial information [3]. These features are built from the successive application of attribute filters, which aim to simplify the image by removing some of its connected components that do not fulfill a given criterion. More precisely, each connected component 
of the level sets is characterized by some attributes, and the simplification (or filtering) is achieved by comparing each attribute value to a predefined threshold. This framework is particularly appealing due to its high efficiency that is ensured by the underlying tree structure (or hierarchical image representation). Processing tree nodes instead of raw pixels leads to a severe decrease in terms of computational cost, allowing this framework to be relevant even for largescale studies. Since their introduction in [3], attribute profiles have been widely used and several recent extensions have been proposed to strengthen their expressivness [4] (e.g. we will use differential APs made of differences between successive values in the profile). We will use these features to characterize $2 \mathrm{D}$ or $3 \mathrm{D}$ structures.

\subsection{Point Clouds and Mathematical Morphology}

The study in [5] is interesting since authors perform mathematical morphology on points in a sound continuous mathematical framework. Their idea consist in computing a surface from the point cloud on which erosion/dilation and associated morphological features can be computed. Though very relevant, this approach can not be applied with multi-echo LiDAR data since surfaces can not be computed. Indeed, in this context we not only extract points related to the boundaries of objects but in some situations (vegetated areas for example), some points can be included inside embedding shapes. Therefore the reconstruction of a single surface for each object is impossible.

For that reason, many authors have tried to compute morphological features on regular 3D data (volumes) defined on voxels. Hence in [6] the authors extend 2D morphological algorithms to 3D voxels for LiDAR point clouds of trees. The density of points in each cell is kept for the value associated with each voxel, as in [7] for astronomical applications. In [8], the same principle is used on binary $3 \mathrm{D}$ images ( 1 if a point is inside the voxel, 0 elsewhere) for the segmentation of facades from urban point clouds, using morphology on DEM with specific filters related to elongation.

In a connected idea, the study in [9] performs segmentation and classification of urban point clouds with super-voxels. Such supervoxels are created on the basis of raw data (intensity, $R, G, B, \ldots$ ) and specific features (mean, variance of raw data, geometric organization, ...). Results are then re-projected by associating the same value to each point inside a voxel.

\subsection{Trees and $3 D$ images}

Max-trees have been introduced for 2D data in [10]. They have then been extended in [11] to 3D voxels on medical images. Such trees are exploited for filtering objects on the basis of inertia attributes. In a similar way, the authors in [12-14] have proposed new 3D geometric attributes to isolate more complex structures (elongation, flatness, sphericity, roundness, sparseness, ... ). Therefore a large variety of studies of been proposed based on mathematical morphology on voxels in the medical image community [15-18].

Surprisingly only few studies have been made in the EO context despite the fact that $3 \mathrm{D}$ data become here more and more important.

\section{METHOD}

Following the bottom of Figure 2, the overall methodology consists in: $i$ ) building a volume composed of regular voxels from the 3D point cloud; ii) computing 3D morphological features on this volume and using these features in a data analysis process (e.g., classification, clustering, filtering, ... ); and iii) projecting the results back into a $2 \mathrm{D}$ grid. These steps are described below.

\subsection{From LiDAR point clouds to $3 D$ rasters}

The voxelization process consists in putting in each voxel of the volume the information related to the 3D points embedded in this voxel. Depending on the application, such information can be a binary map related to the presence/absence of points, the number of LiDAR points into the voxel, the average/standard deviation of associated intensities, the average/standard deviation of associated elevations, the label in majority (for 3D labels), ...

\subsection{D hierarchical representations}

Construcing min- and max-trees is then achieved following methods introduced in Section 1.3. We consider here a 26-connectivity (i.e. two voxels are neighbors if they share a plane, edge or vertex).

\subsection{AP on 3D data}

As already stated, we illustrate here the voxelization process with attribute profiles (APs) which have been introduced in Section 1.1. For the sake of simplicity, we associate to each voxel a scalar value on which an ordering relation (required to build the tree) can be straightforwardly defined. We consider several attributes: intensity distribution, volume, surface, bounding box, and moment-based. These attributes are then compared to a set of thresholds to filter the max-tree and then to generate the APs.

\subsection{From 3D to 2D maps}

In order to project the $3 \mathrm{D}$ information (defined on $(X, Y, Z)$ ) in $2 \mathrm{D}$ grids (defined on $(X, Y)$ ), one needs to transform volumes into maps and to summarize the vertical information in axis $Z$. Different strategies are possible depending on the data and are listed below:

\section{Elevation rules:}

1. Surface model: points with higher altitude in the vertical dimension (i.e. higher objects as trees or buildings appear first);

2. Terrain cover: points with lower altitude in the vertical dimension (i.e. ground and streets appear first);

\section{Label rules:}

3. Priority rule: labels are kept depending on their priority (e.g. cars first, then trees, then roads, ...);

4. Majority rule: the majority label in the vertical axis is kept;

Value rules:

5. Average/standard deviation of each value in the vertical axis.

\section{EXPERIMENTS}

\subsection{Dataset}

Experiments have been conduced on the Paris Lille Dataset [19], which is made of a very high spatial resolution point cloud (approx $1000 \sim 2000$ point per $\mathrm{m}^{2}$ ) available online. As the laser scan is performed from a car in the middle of the street, the density of points depends mainly on the distance with respect to the car but not on the nature of objects. Therefore, this quantity can not be taken into account. As for the intensity, which is usually of poor quality in such data, most of associated values are constant. Similarly to all LiDAR data, the intensity value also depends on the distance of objects w.r.t the scanner. Average intensities and elevations are the 


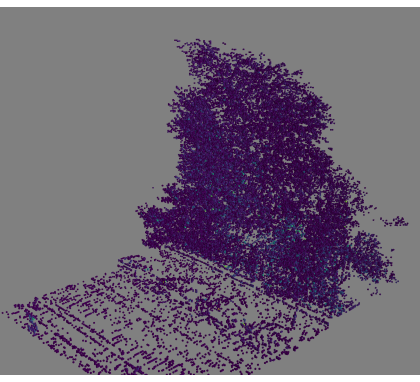

(a) $0<\Lambda \leq 10$

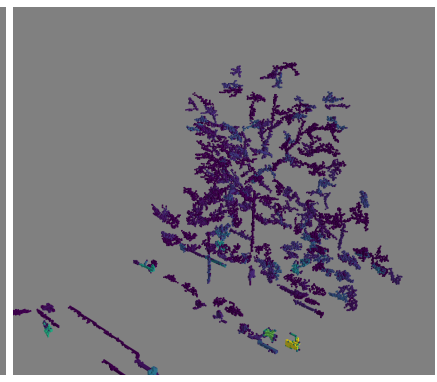

(b) $50<\Lambda \leq 100$

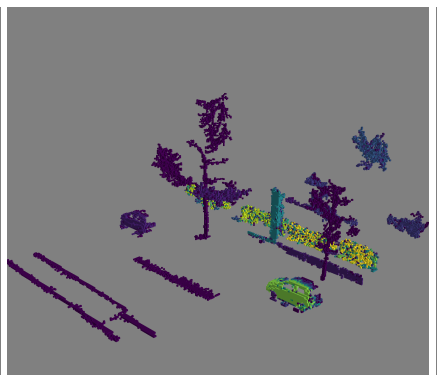

(c) $500<\Lambda \leq 1000$

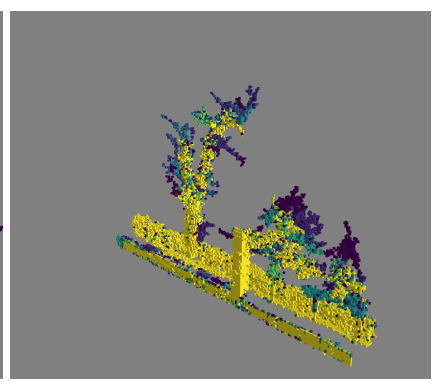

(d) $10000<\Lambda \leq 20000$

Fig. 3: Visualisation of the hierarchical representation involved with APs using the derivative of attribute profiles (DAPs).

two information kept in each voxel. The scene has been classified in 5 objects: cars, street, fences, trees and urban furniture.

\subsection{Experimental setup}

In each experiment, the volumes have been computed on a voxel grid of $10 \mathrm{~cm}^{3}$ spatial resolution. APs (2D or 3D) have been computed with area and volume criteria. The thresholds have been empirically set to $\Lambda_{V}=\left\{10,50,100,200,500,1000,1 \times 10^{4}, 2 \times 10^{4}, 1 \times 10^{5}\right\}$ for volume and $\Lambda_{A}=\left(\sqrt[3]{\Lambda_{V}}\right)^{2}$ for area. A first set of experiments related to $3 \mathrm{D}$ classification (with generic name $T D$ for "TriDimensions") has been conducted to evaluate the benefits of 3D AP on 3D data: i) with intensity (noted $T D_{i}$ ); ii) with intensity and elevation $\left(T D_{i e}\right)$; iii) with APs built on intensity $\left(T D_{A i}\right)$; iv) with APs built on intensity and elevation $\left(T D_{A i e}\right)$.

Then, a second set of experiments is devoted to the evaluation of 3D AP features for 2D classification (with generic name $B D$ for "Bi-Dimension"). To this end, we keep the best 3D features for 3D classifications (issued from the first series) and we compare their performances in 2D and 3D versions for 2D classification. 3D labels are reprojected on 2D grids based on the majority label. Classification have been performed using Random Forest technique. Some illustrations of hierarchical representations using Differential-AP with various thresholds of volumes are visible in Figure 3. Validation criteria rely on Overall Accuracy and Cohen's Kappa coefficient. During $3 \mathrm{D}$ to $2 \mathrm{D}$ projection, the majority label in the vertical component has been kept. In practice, training and validation data have been taken from various tiles of the dataset.

\subsection{Results}

\subsubsection{Voxel grid classification}

Evaluation measures for 3D classification are reported in Table 1. One can see that the elevation feature enables to improve the classification with intensity only, and that the use of APs enables to improve the overall accuracy of the classification (the overall accuracy and kappa of $T D_{A i}$-resp. $T D_{A i e}$ is higher than the ones of experiment $T D_{i}$-resp. $T D_{i e}$ ). The best combination $T D_{A i e}$ provides really satisfying results and an illustration of classification is depicted in Figure 4. This first set of experiments justifies the use of APs and Random Forest for 3D data classification. Let us now evaluate the benefits of $3 \mathrm{D}$ features for $2 \mathrm{D}$ classification.

\begin{tabular}{l|l|rr}
\hline & Description method & OA $(\%)$ & $\kappa(\times 100)$ \\
\hline \hline$T D_{i}$ & Intensity & 75.28 & 48.80 \\
$T D_{i e}$ & Intensity and elevation & 91.10 & 82.92 \\
$T D_{A i}$ & Intensity APs & 78.82 & 58.63 \\
$T D_{A i e}$ & Intensity APs and elevation & $\mathbf{9 3 . 6 6}$ & $\mathbf{8 7 . 9 9}$ \\
\hline
\end{tabular}

Table 1: Overall accuracy (OA) and Cohen's Kappa coefficient $(\kappa)$ in $3 \mathrm{D}$ voxel grid space, with and without AP.

\begin{tabular}{l|l|rr}
\hline & Description method & OA $(\%)$ & $\kappa(\times 100)$ \\
\hline \hline$B_{2 D \text { Aie }}$ & Intensity and elevation APs 2D & 98.25 & 96.61 \\
BD 3 3D Aie & Intensity APs and elevation 3D to 2D & $\mathbf{9 9 . 3 6}$ & $\mathbf{9 8 . 7 6}$ \\
\hline
\end{tabular}

Table 2: Overall accuracy (OA) and Cohen's Kappa coefficient $(\kappa)$ in $2 \mathrm{D}$ classification with the best features issued from Table 1 in 2D and $3 \mathrm{D}$ versions.

\subsubsection{Pixel grid classification}

In this second series of experiments, 2D classifications have been performed on the basis of the best 3D features in $2 \mathrm{D}$ and $3 \mathrm{D}$ versions. More precisely, we have computed 2D classification with APs computed on 2D intensity and elevation (noted $B D_{2 D A i e}$ ) and 2D classification with APs computed on 3D intensity and elevation and $2 \mathrm{D}$ reprojected in the pixel grid (noted $B D_{3 D A i e}$ ). Quantitative results are given in Table 2. It is interesting to observe that as expected the generation of 2D maps on the basis of 3D features enables to improve the quality (both in terms of overall accuracy and kappa) of the 2D classifications, which is a really interesting property. An illustration is visible on Figure 5.

\section{CONCLUSION}

In this paper, we have proposed to classify LiDAR data on the basis of 3D features. Unlike most studies that rasterize the 3D point cloud in a first step, we have suggested to rather voxelize the 3D point cloud, before computing some hierarchical features on this volume and perfoming a subsequent supervised classification. The rasterization is performed only in a final step. This process has the advantage to keep the 3D information of structures along the process instead of removing this information through early rasterization. The experimental results on 3D urban LiDAR data have quantitatively demonstrated the relevance of such an approach. 


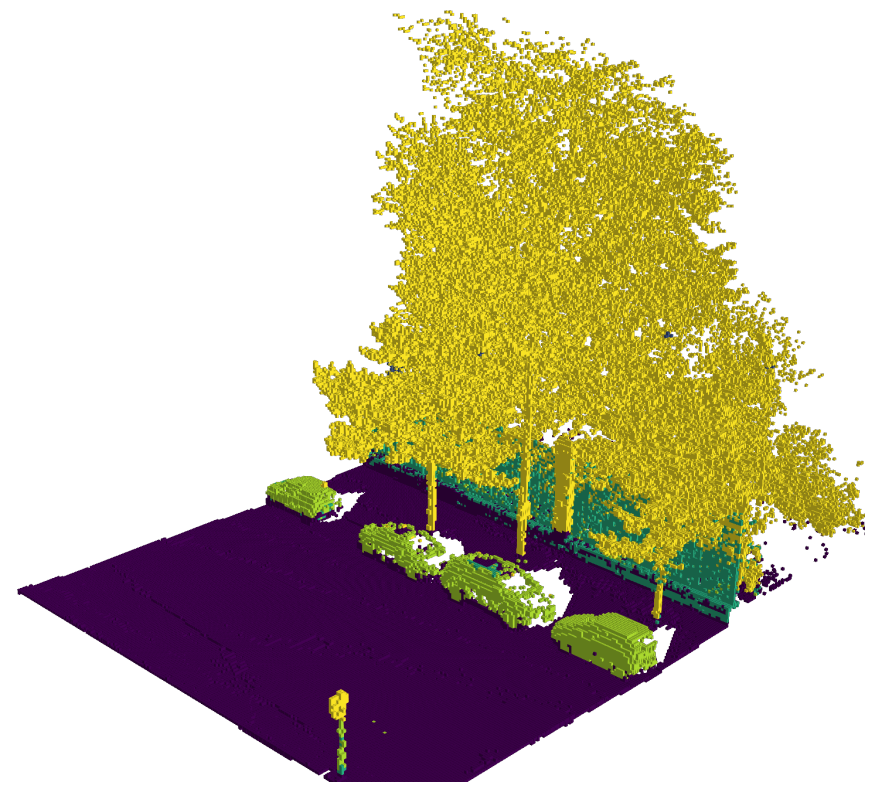

Fig. 4: Classification of the scene in the 3D voxel grid space using intensity data with APs and elevation information.

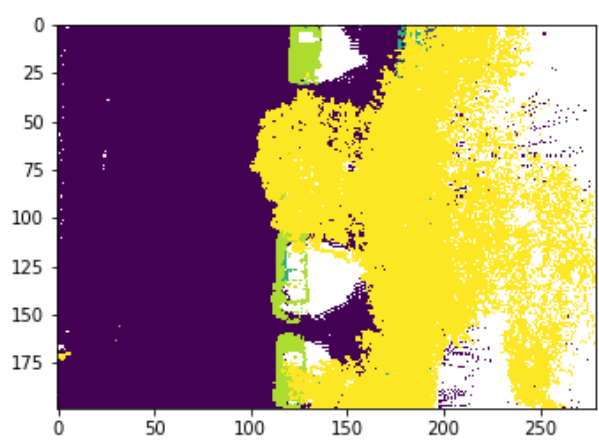

Fig. 5: 2D classification result obtained from Figure 4 voxel grid reprojected into the $2 \mathrm{D}$ map space using the surface rule (see 2.4).

\section{ACKNOWLEDGMENT}

The authors would like to thank the authors of Paris Dataset [19] for providing this public dataset.

\section{REFERENCES}

[1] A. Serna and B. Marcotegui, "Detection, segmentation and classification of $3 \mathrm{~d}$ urban objects using mathematical morphology and supervised learning," ISPRS J., vol. 93, pp. 243-255, 2014.

[2] F. Guiotte, S. Lefevre, and T. Corpetti, "Rasterization strategies for airborne LiDAR classification using attribute profiles," in JURSE, 2019.

[3] M. Dalla Mura, J.A. Benediktsson, B. Waske, and L. Bruzzone, "Morphological attribute profiles for the analysis of very high resolution images," IEEE TGRS, vol. 48, no. 10, pp. 37473762, 2010.

[4] M.T. Pham, E. Aptoula, S. Lefevre, and L. Bruzzone, "Recent Developments from Attribute Profiles for Remote Sensing Image Classification," in ICPRAI, 2018.

[5] S. Calderon and T. Boubekeur, "Point Morphology," ACM $T o G, 2014$.

[6] B. Gorte and N. Pfeifer, "Structuring laser-scanned trees using 3d mathematical morphology," ISPRS Archives, vol. 35, no. B5, pp. 929-933, 2004.

[7] B.J. Ferdosi, H. Buddelmeijer, S. Trager, M.H.F. Wilkinson, and J.B.T.M. Roerdink, "Finding and visualizing relevant subspaces for clustering high-dimensional astronomical data using connected morphological operators," in IEEE Symposium on Visual Analytics Science and Technology, 2010, pp. 35-42.

[8] A. Serna, B. Marcotegui, and J. Hernández, "Segmentation of Façades from Urban 3d Point Clouds using Geometrical and Morphological Attribute-based Operators," ISPRS IJGI, vol. 5, no. 1, 2016.

[9] A. Aijazi, P. Checchin, and L. Trassoudaine, "Segmentation Based Classification of 3d Urban Point Clouds: A Super-Voxel Based Approach with Evaluation," Remote Sensing, vol. 5, no. 4, pp. 1624-1650, 2013.

[10] P. Salembier, A. Oliveras, and L. Garrido, "Antiextensive connected operators for image and sequence processing," IEEE TIP, vol. 7, no. 4, pp. 555-570, 1998.

[11] M.H.F. Wilkinson and M.A. Westenberg, "Shape Preserving Filament Enhancement Filtering," in MICCAI, 2001, vol. 2208.

[12] M.A. Westenberg, J.B.T.M. Roerdink, and M.H.F. Wilkinson, "Volumetric Attribute Filtering and Interactive Visualization Using the Max-Tree Representation," IEEE TIP, vol. 16, no. 12, pp. 2943-2952, 2007.

[13] F.N. Kiwanuka, G.K. Ouzounis, and M.H.F. Wilkinson, "Surface-area-based attribute filtering in 3d," in ISMM, 2009.

[14] F.N. Kiwanuka and M.H.F. Wilkinson, "Radial Moment Invariants for Attribute Filtering in 3d," in WADGMM, 2012.

[15] A. Dufour, O. Tankyevych, B. Naegel, H. Talbot, C. Ronse, J. Baruthio, P. Dokládal, and N. Passat, "Filtering and segmentation of 3d angiographic data: Advances based on mathematical morphology," Medical Image Analysis, vol. 17, no. 2, pp. 147-164, 2013.

[16] E. Grossiord, H. Talbot, N. Passat, M. Meignan, P. Terve, and L. Najman, "Hierarchies and shape-space for pet image segmentation," in ISBI, 2015, pp. 1118-1121.

[17] H. Urien, I. Buvat, N. Rougon, M. Soussan, and I. Bloch, "Brain Lesion Detection in 3d PET Images Using Max-Trees and a New Spatial Context Criterion," in ISMM, 2017.

[18] F.J.A. Padilla, B. Romaniuk, B. Naegel, S. Servagi-Vernat, D. Morland, D. Papathanassiou, and N. Passat, "Hierarchical forest attributes for multimodal tumor segmentation on FDGPET/contrast-enhanced CT," in ISBI, 2018, pp. 163-167.

[19] X. Roynard, J.-E. Deschaud, and F. Goulette, "Paris-Lille-3d: a large and high-quality ground truth urban point cloud dataset for automatic segmentation and classification," ArXiv, 2017. 